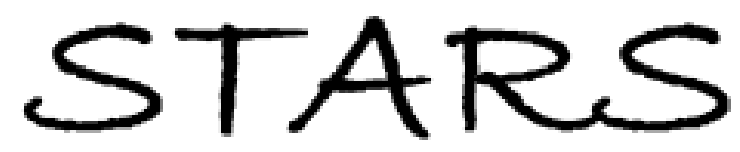

University of Central Florida

STARS

$1-1-2006$

\title{
Role of trivalent La and Nd dopants in lattice distortion and oxygen vacancy generation in cerium oxide nanoparticles
}

\author{
Swanand Patil \\ University of Central Florida \\ Sudipta Seal \\ University of Central Florida \\ Yu Guo \\ University of Central Florida \\ Alfons Schulte \\ University of Central Florida \\ John Norwood
}

Find similar works at: https://stars.library.ucf.edu/facultybib2000

University of Central Florida Libraries http://library.ucf.edu

This Article is brought to you for free and open access by the Faculty Bibliography at STARS. It has been accepted for inclusion in Faculty Bibliography 2000s by an authorized administrator of STARS. For more information, please contactSTARS@ucf.edu.

\section{Recommended Citation}

Patil, Swanand; Seal, Sudipta; Guo, Yu; Schulte, Alfons; and Norwood, John, "Role of trivalent La and Nd dopants in lattice distortion and oxygen vacancy generation in cerium oxide nanoparticles" (2006).

Faculty Bibliography 2000s. 7877.

https://stars.library.ucf.edu/facultybib2000/7877

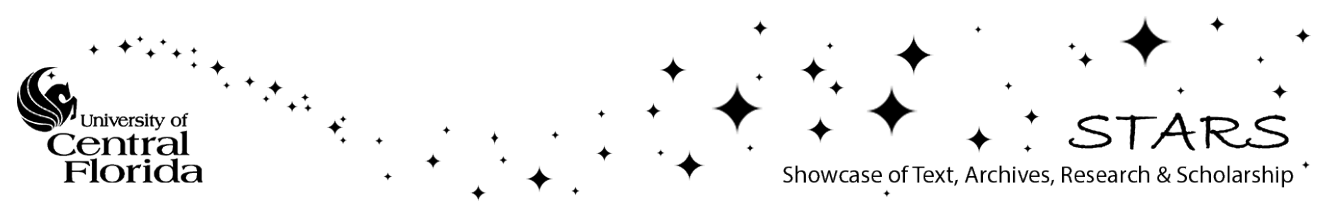




\section{Role of trivalent La and Nd dopants in lattice distortion and oxygen vacancy generation in cerium oxide nanoparticles}

Cite as: Appl. Phys. Lett. 88, 243110 (2006); https://doi.org/10.1063/1.2210795

Submitted: 04 February 2006. Accepted: 10 May 2006. Published Online: 14 June 2006

Swanand Patil, Sudipta Seal, Yu Guo, Alfons Schulte, and John Norwood

ARTICLES YOU MAY BE INTERESTED IN

Cerium oxide nanoparticles: Size-selective formation and structure analysis

Applied Physics Letters 80, 127 (2002); https://doi.org/10.1063/1.1430502

Size dependency variation in lattice parameter and valency states in nanocrystalline cerium oxide

Applied Physics Letters 87, 133113 (2005); https://doi.org/10.1063/1.2061873

Raman and $\mathrm{x}$-ray studies of $\mathrm{Ce}_{1-\chi} \mathrm{RE}_{\chi} \mathrm{O}_{2-y}$, where $\mathrm{RE}=\mathrm{La}, \mathrm{Pr}, \mathrm{Nd}$, Eu, $\mathrm{Gd}$, and $\mathrm{Tb}$

Journal of Applied Physics 76, 2435 (1994); https://doi.org/10.1063/1.357593
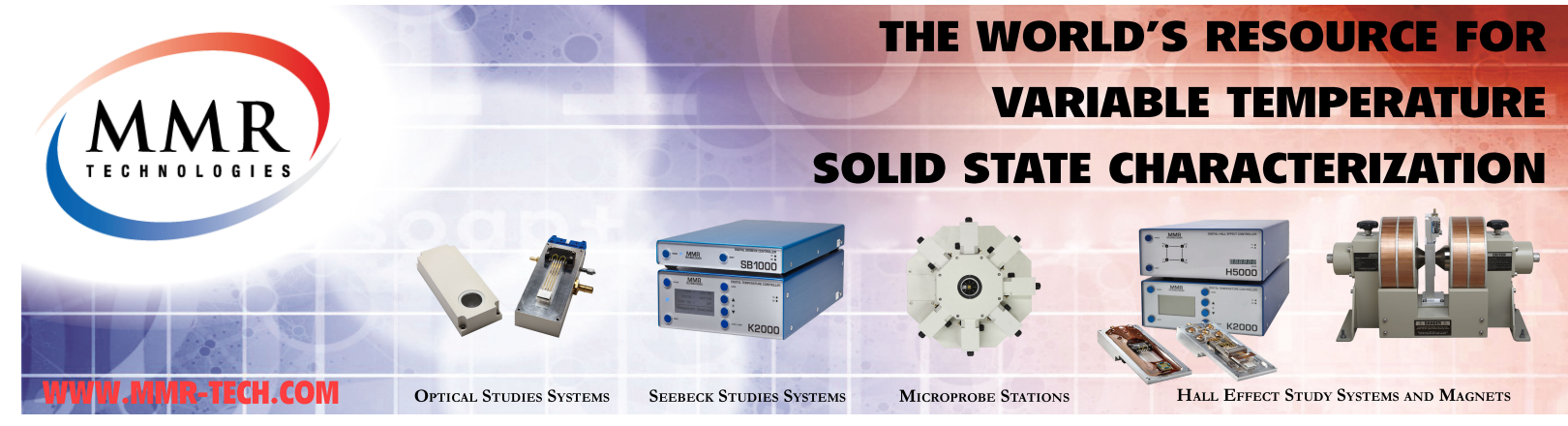


\title{
Role of trivalent La and Nd dopants in lattice distortion and oxygen vacancy generation in cerium oxide nanoparticles
}

\author{
Swanand Patil and Sudipta Seal ${ }^{\mathrm{a}}$ \\ Advanced Materials Processing and Analysis Center (AMPAC), Nanoscience Technology Center and \\ Mechanical Materials and Aerospace Engineering (MMAE), University of Central Florida, 4000 Central \\ Florida Boulevard, Orlando, Florida 32816 \\ Yu Guo and Alfons Schulte \\ Department of Physics, University of Central Florida, 4000 Central Florida Boulevard, Orlando, Florida \\ 32816 \\ John Norwood ${ }^{\text {b) }}$ \\ Department of Civil and Environmental Engineering, Princeton University, Princeton, New Jersey 08544
}

(Received 4 February 2006; accepted 10 May 2006; published online 14 June 2006)

\begin{abstract}
Nanocrystalline $\mathrm{Ce}_{1-x} \mathrm{RE}_{x} \mathrm{O}_{2-y}(\mathrm{RE}=\mathrm{La}$ and $\mathrm{Nd})$ powders were synthesized with a controlled size in the range of 3-5 $\mathrm{nm}$ using microemulsion technique. The nanoceria retains its cubic fluorite structures for the complete doping range. X-ray diffraction and Raman spectroscopic studies showed that the lattice distortion increased with the doping amount and was found to be higher for La-doped samples compared to the Nd doping. Spatial correlation model used for Raman analysis suggested increased defect concentration for the doped samples compared to nanoceria. () 2006 American Institute of Physics. [DOI: 10.1063/1.2210795]
\end{abstract}

Cerium oxide is of interest due to its wide variety of applications such as an electrolyte material in solid oxide fuel cells, ${ }^{1}$ for gas sensors, ${ }^{2}$ ultraviolet absorbents, ${ }^{3}$ and high temperature oxidation resistant coatings. ${ }^{4}$ Many of these applications can benefit from monodisperse $\mathrm{CeO}_{2}$ nanoparticles due to properties confined to their nanostructure such as blueshift in ultraviolet absorption spectrum, ${ }^{3}$ shifting and broadening in Raman allowed modes, ${ }^{5}$ and lattice expansion. ${ }^{6,7}$ Our earlier studies have shown that lattice constant of nanocrystalline cerium oxide particles increases with decreasing particle size. ${ }^{6}$ Also the Raman studies of $\mathrm{CeO}_{2}$ nanoparticles have demonstrated that the mode near $464 \mathrm{~cm}^{-1}$ shifts to progressively lower energies and the line shape of this feature gets progressively asymmetric as the particle size gets smaller. ${ }^{5,8}$ The size dependence has been attributed to the inhomogeneous strain broadening associated with the dispersion in particle size and phonon confinement.

Recently, lot of interest has been generated in nanoceria for various catalytic applications due to its ability to easily absorb and release oxygen. ${ }^{9}$ The ability to store oxygen is a result of cerium's ability to change valence states and the presence of intrinsic $\mathrm{O}$ vacancies in the $\mathrm{CeO}_{2}$ lattice. Addition of trivalent elements in the $\mathrm{CeO}_{2}$ crystal lattice also causes formation of $\mathrm{O}$ vacancies by replacing one $\mathrm{Ce}^{4+}$ ion for every two trivalent ions in the $\mathrm{CeO}_{2}$ lattice. These vacancies increase $\mathrm{O}$ diffusion and thereby increase the ease with which the material can absorb and release $\mathrm{O}$.

Although the doped $\mathrm{CeO}_{2}$ systems have been investigated, the doping effect in ceria nanoparticles with well characterized size is not done previously. The doping of ceria at that small size can be very beneficial to further improve its catalytic properties. The present article demonstrates the doping effect of monodisperse cerium oxide nanoparticles

\footnotetext{
${ }^{\text {a) }}$ Author to whom correspondence should be addressed; electronic mail: sseal@mail.ucf.edu

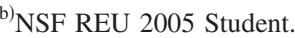

with varying amounts of $\mathrm{La}$ and $\mathrm{Nd}$ in the controlled particle size of $3-5 \mathrm{~nm}$.

The doped nanoparticles were synthesized using microemulsion process with cerium nitrate, lanthanum nitrate, and neodymium nitrate as the precursors and ammonium hydroxide as a coprecipitating agent. All the chemicals were obtained from Aldrich Chemicals Company Inc. and used without further purification. The precursors were mixed with different atomic ratios, $N_{\mathrm{RE}} / N_{\mathrm{Ce}}$, where $\mathrm{RE}=\mathrm{La}$ or $\mathrm{Nd}$. The dopant ( $\mathrm{La}$ or $\mathrm{Nd}$ ) percentage was determined by the formula at. \% dopant $=\left(100 N_{\mathrm{RE}}\right) /\left(N_{\mathrm{Ce}}+N_{\mathrm{RE}}\right)$. The details of the samples are given in Table I. The synthesis procedure was the same as described elsewhere. ${ }^{10}$ The nanoparticle powders obtained by washing the precipitates several times with acetone and water were characterized using high resolution transmission electron microscopy (HRTEM), x-ray diffraction (XRD), and Raman spectroscopy.

The HRTEM images were obtained with Philips (Tecnai series) transmission electron microscope operated at $300 \mathrm{keV}$. The bright field HRTEM micrographs of all the doped samples in Fig. 1 exhibit near-spherical $3-5 \mathrm{~nm}$ particles with a uniform particle size distribution. The selected area diffraction patterns shown in the inset of the micro-

TABLE I. Details of the nanoceria samples doped with $\mathrm{La}$ and $\mathrm{Nd}$.

\begin{tabular}{|c|c|c|c|}
\hline \multirow[b]{2}{*}{ Sample } & \multicolumn{2}{|c|}{ Atomic ratio of dopants used for synthesis } & \multirow{2}{*}{$\begin{array}{c}\text { Dopant amount } \\
\text { (at. \%) }\end{array}$} \\
\hline & $N_{\mathrm{La}} / N_{\mathrm{Ce}}$ & $N_{\mathrm{Nd}} / N_{\mathrm{Ce}}$ & \\
\hline $\mathrm{CeO}_{2}-0$ & $\cdots$ & $\cdots$ & 0 \\
\hline $\mathrm{CeO}_{2}-1$ & $5 / 100$ & $\cdots$ & 4.76 \\
\hline $\mathrm{CeO}_{2}-2$ & $10 / 100$ & $\ldots$ & 9.09 \\
\hline $\mathrm{CeO}_{2}-3$ & $20 / 100$ & $\cdots$ & 16.67 \\
\hline $\mathrm{CeO}_{2}-4$ & $\cdots$ & $5 / 100$ & 4.76 \\
\hline $\mathrm{CeO}_{2}-5$ & $\cdots$ & $10 / 100$ & 9.09 \\
\hline $\mathrm{CeO}_{2}-6$ & $\cdots$ & $20 / 100$ & 16.67 \\
\hline
\end{tabular}



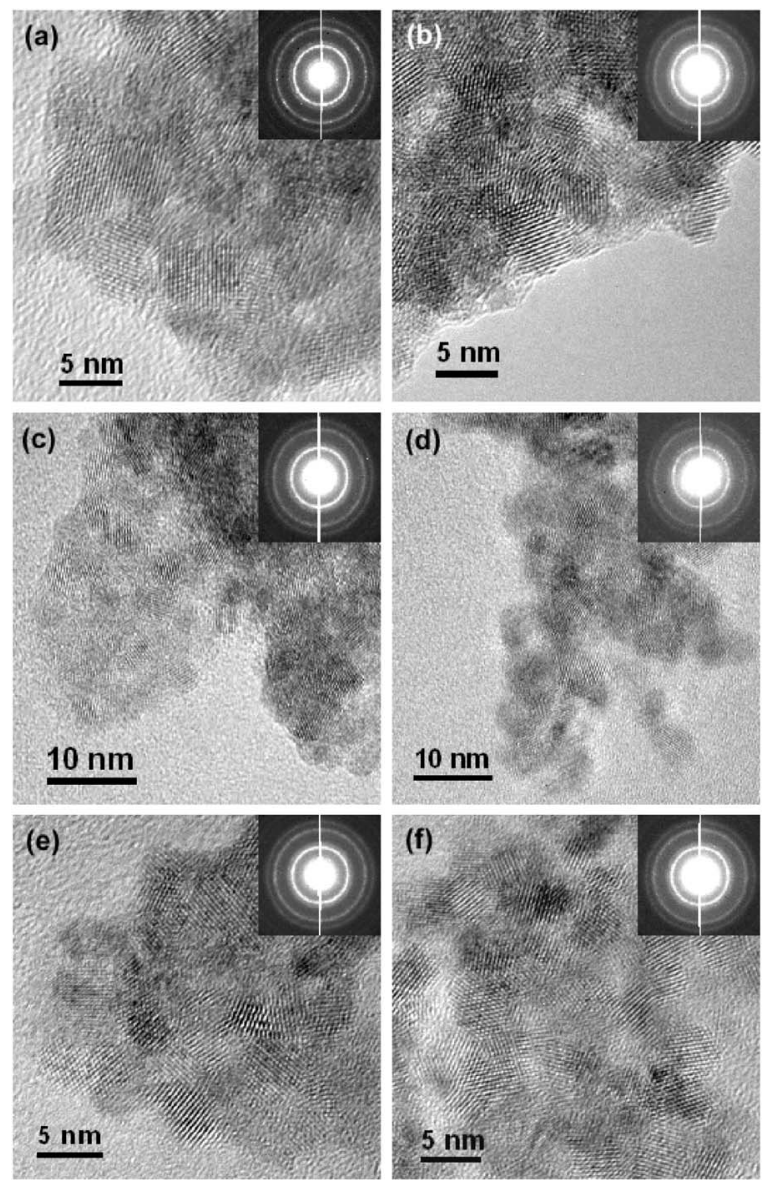

FIG. 1. HRTEM images of doped ceria samples (a) $\mathrm{CeO}_{2}-1$, (b) $\mathrm{CeO}_{2}-2$, (c) $\mathrm{CeO}_{2}-3$, (d) $\mathrm{CeO}_{2}-4$, (e) $\mathrm{CeO}_{2}-5$, and (f) $\mathrm{CeO}_{2}-6$.

graphs prove the nanocrystalline nature of the synthesized powders with fluorite crystal structure.

The crystal structure was determined with x-ray diffraction (Rigaku model) using $\mathrm{Cu} K \alpha_{1}$ radiation. The data were recorded at a scan rate of $0.2^{\circ} / \mathrm{min}$. The XRD patterns of all the samples along with microceria (obtained from Johnson Matthey) are shown in Fig. 2(a). The broadened peaks in all the diffractograms confirm the nanocrystalline nature of the synthesized powder samples and the peaks can be indexed to the fluorite structure of cerium oxide. The absence of the additional peaks related to $\mathrm{La}_{2} \mathrm{O}_{3}$ or $\mathrm{Nd}_{2} \mathrm{O}_{3}$ in La-doped and $\mathrm{Nd}$-doped ceria samples, respectively, indicates the formation of single phase $\mathrm{Ce}_{1-x} \mathrm{RE}_{x} \mathrm{O}_{2-y}$-type solid solution for the full doping range.

The lattice constant of all the samples was measured by fitting the peaks using PEAKFIT (version 4.0) software. Figure 2(b) shows the increase in the lattice constant with the dopant concentration. This means that doping affects the crystal structure of nanoceria. The lattice constant shifts shown in Fig. 2(b) can be directly correlated with the ionic size of the dopant. As the ionic radius of $\mathrm{La}^{3+}(0.122 \mathrm{~nm})$ is larger than that of $\mathrm{Nd}^{3+}(0.115 \mathrm{~nm})$, the lattice constant is larger for all the La-doped samples $\left(\mathrm{CeO}_{2}-1-\mathrm{CeO}_{2}-3\right)$ compared to the corresponding Nd-doped samples $\left(\mathrm{CeO}_{2}-4-\mathrm{CeO}_{2}-6\right)$. The data obtained in Fig. 2(b) can be fitted by a straight line showing a linear trend in lattice expansion with the doping concentration. The slopes of the lines can be found to be 0.0297 and $0.0182 \mathrm{~nm} /$ atomic fraction for $\mathrm{La}$ and $\mathrm{Nd}$ dopings, respectively.

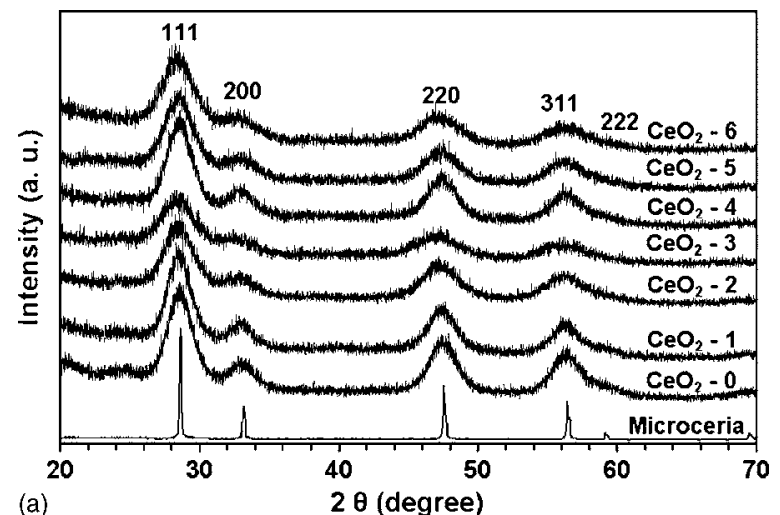

(a)

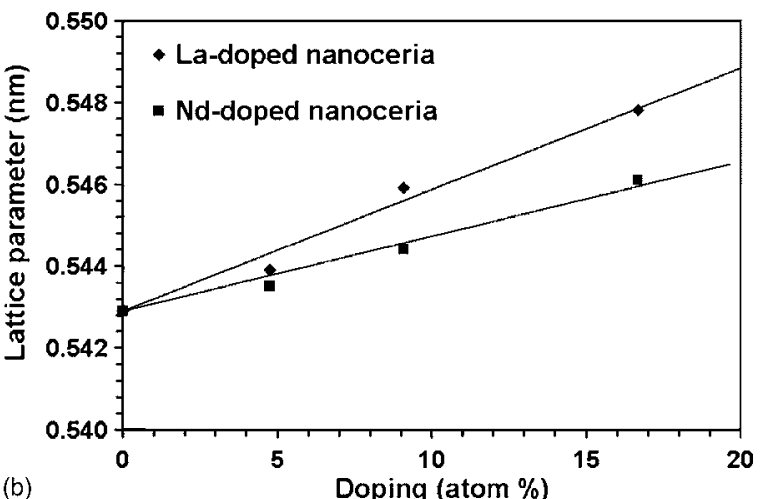

FIG. 2. (a) XRD spectra of the nanoceria samples. (b) Lattice parameter variation of the nanoceria samples as a function of doping amount.

Raman scattering is an excellent, nondestructive, and rapid analysis technique for investigating the electronic and phonon structure of materials. The different symmetries involved govern whether vibrations are Raman active and appear in the spectra, while changes in lattice spacing and chemical environment shift band frequencies. Raman spectra for the synthesized samples were measured with a Horiba Jobin Yvon LabRam IR micro-Raman system with a spatial resolution of $2 \mu \mathrm{m}$. A helium-neon laser provided excitation at $633 \mathrm{~nm}$ with a power of $3 \mathrm{~mW}$.

Cerium oxide has a cubic fluorite-type structure and belongs to the $\mathrm{O}_{h}^{5}(\mathrm{Fm} 3 \mathrm{~m})$ space group. This structure has six optical-phonon branches, which yield three zone-center frequencies. These frequencies are 272,465 , and $595 \mathrm{~cm}^{-1}$, corresponding to the doubly degenerated TO mode, the triply degenerate Raman-active mode, and the nondegenerate LO mode, respectively. The triply degenerate Raman-active mode frequency can be directly detected by Raman measurements, whereas the TO and LO frequencies are given indirectly by fits to infrared reflectivity. ${ }^{11}$

The presence of $\mathrm{RE}^{3+}$ in the $\mathrm{CeO}_{2}$ lattice deforms the structure and can be detected by Raman analysis. Figure 3(a) shows the measured Raman spectra for the La- and Nddoped nanoceria samples, along with the spectra for microceria and nanoceria. The shift in the Raman mode for nanoceria $\left(458 \mathrm{~cm}^{-1}\right)$ compared to the microceria $\left(464 \mathrm{~cm}^{-1}\right)$ proves the particle size effect which was seen earlier. ${ }^{5,6}$ The peak frequencies for the single Raman line were determined by fitting the data to a Lorentzian line shape using PEAKFIT (version 4.0) software. Figure 3(c) shows the Raman allowed mode frequency shifts measured for all the samples. More peak shift (to lower energies) for the La-doped samples compared to Nd-doped samples indicates more lattice distortion 


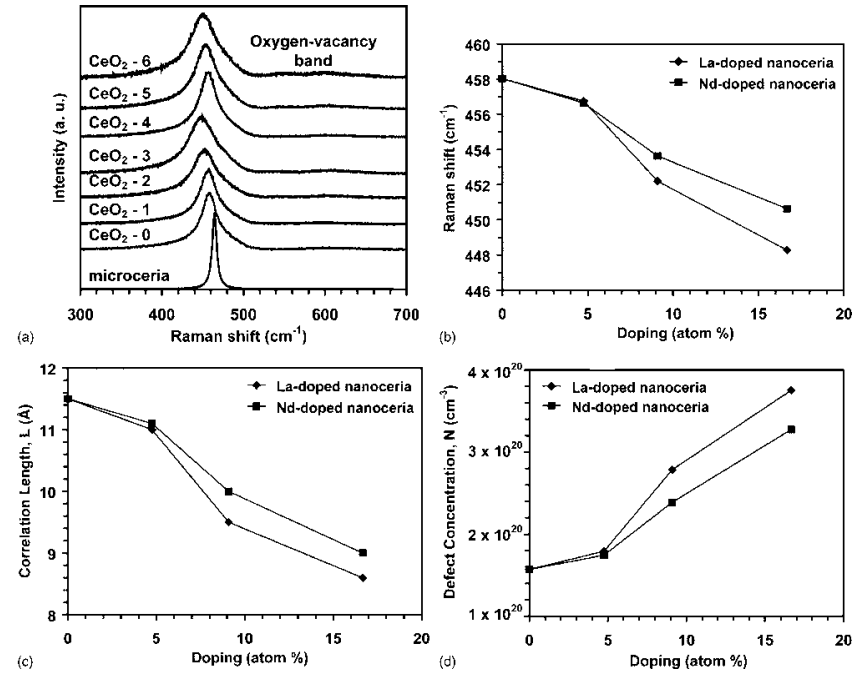

FIG. 3. (a) Raman spectra of all the nanoceria samples. (b) The Raman peak shift, (c) the correlation length obtained from spatial correlation model given in Eq. (1), and (d) oxygen vacancy concentration calculated using Eq. (5) as a function of doping amount.

in the case of La-doped samples. The increasing lattice constant with doping amount as measured by XRD explains the Raman shift well.

With increasing dopant concentration the line shape of the Raman allowed mode gets progressively broader and asymmetric. The lattice disorder induced by the substitution of $\mathrm{RE}^{3+}$ ions causes this shape change in the Raman line which can be studied using the spatial correlation model. ${ }^{8,12}$ According to this model, the Raman line intensity $I(w)$ at frequency $w$ can be written as

$$
I=\int_{0}^{1} \exp \left(\frac{-q^{2} L^{2}}{4}\right) \frac{d^{3} q}{[w-w(q)]^{2}+\left(\Gamma_{0} / 2\right)},
$$

where $q$ is the wave vector expressed in units of $2 \pi / a$ ( $a$ is the lattice constant). $\Gamma_{0}$ is the full width at half maximum (FWHM) of Raman line of large grain sized material $\left(6.9 \mathrm{~cm}^{-1}\right.$ observed for microceria sample). The expression $\exp \left(-q^{2} L^{2} / 4\right)$ represents a Gaussian spatial correlation function with parameter $L$ as correlation length. For dispersion $w(q)$, we fitted the phonon dispersion determined from rigidion model ${ }^{11}$ with analytical model relations

$$
\begin{aligned}
& {[w(q)]_{1}=506-44.105 \cos (\pi q),} \\
& {[w(q)]_{2}=462.5-175 q^{2},} \\
& {[w(q)]_{3}=377.1+86.58 \cos (\pi q) .}
\end{aligned}
$$

As the triple degeneracy of the Raman mode at $464 \mathrm{~cm}^{-1}$ is removed away from $q=0$, Eq. (1) was summed over three equally weighted branches of phonon dispersion given by Eqs. (2)-(4).

The Raman spectrum line-shape fit was performed using the spatial correlation model explained above with only correlation length $L$ as a fitting parameter. The correlation length was found to be $10 \mathrm{~nm}$ for microceria while it decreases gradually from $1.15 \mathrm{~nm}$ for pure nanoceria to 0.86 and $0.90 \mathrm{~nm}$ (less than two lattice constants) for 16.67 at. \% La-doped and Nd-doped samples, respectively. Figure 3(c) shows the determined correlation length for doped nanoceria as a function of doping concentration.
The correlation length $L$ corresponds to the spheres with diameters equal to the distance between two next oxygen vacancies and can be used to determine the defect concentration $N$ as ${ }^{8}$

$$
N=\frac{3}{4 \pi L^{3}} \text {. }
$$

Figure 3(d) presents the defect concentration calculated from the correlation length as given in Eq. (5). The particle size effect increases the defect concentration approximately three orders of magnitude from $10^{17} \mathrm{~cm}^{-3}$ for microceria to $10^{20} \mathrm{~cm}^{-3}$ for nanoceria and then gradually increases with dopant concentration. When $\mathrm{Ce}^{4+}$ ions are substituted by $\mathrm{RE}^{3+}$ ions, the oxygen vacancies are introduced into the fluorite lattice by partial reduction of $\mathrm{Ce}^{4+}$ to $\mathrm{Ce}^{3+}$ so as to maintain the electric neutrality. This reduction process can be given as follows:

$$
\begin{aligned}
& \mathrm{RE}_{2} \mathrm{O}_{3} \stackrel{\mathrm{CeO}_{2}}{\longrightarrow} 2 \mathrm{RE}_{\mathrm{Ce}}^{\prime}+V_{\mathrm{O}}^{\prime \prime}+3 \mathrm{O}_{\mathrm{O}}^{x}, \\
& 2 \mathrm{Ce}_{\mathrm{Ce}}^{x}+\mathrm{O}_{\mathrm{O}}^{x}=2 \mathrm{Ce}_{\mathrm{Ce}}^{\prime}+V_{\mathrm{O}}^{\prime \prime}+\frac{1}{2} \mathrm{O}_{2}(g),
\end{aligned}
$$

where $\mathrm{RE}=\mathrm{La}$ or $\mathrm{Nd}$.

Additionally, there is a broad band in the range of $540-640 \mathrm{~cm}^{-1}$ which increases with doping amount. A mathematical model based on Green's function was used to attribute this feature to oxygen vacancies. ${ }^{13}$

In conclusion, cerium oxide nanoparticles doped with varying dopant amounts were synthesized using microemulsion process. The size-dependent lattice expansion, induced by increased oxygen vacancies, in $\mathrm{CeO}_{2}$ nanoparticles can be further increased by the trivalent dopants without altering the particle size. Due to larger ionic radius, La doping generates more defects in the nanoceria lattice compared to Nd doping. Such doped ceria nanoparticles can be useful for improved catalytic applications.

This research is funded by NSF CTS 0350572, EEC 0453436, BES 0541516, NASA Glenn NAG 32751, ONR Young Investigator Award No. ONR N000140210591, DOE SBIR Phase IDE-FG02-04ER83994, and NSF DMR 0421253 .

${ }^{1}$ K. Eguchi, T. Setoguchi, T. Inoue, and H. Arai, Solid State Ionics 52, 165 (1992).

${ }^{2}$ P. Jasinski, T. Suzuki, and H. U. Anderson, Sens. Actuators B 95, 73 (2003).

${ }^{3}$ S. Tsunekawa, R. Sivamohan, T. Ohsuna, A. Kasuya, H. Takahashi, and K. Tohji, Rare Earths '98 (Trans Tech, Switzerland, 1999), Vols. 315-317, pp. 439-445.

${ }^{4}$ S. Patil, S. C. Kuiry, and S. Seal, Proc. R. Soc. London, Ser. A 460, 3569 (2004).

${ }^{5}$ J. E. Spanier, R. D. Robinson, F. Zheng, S. W. Chan, and I. P. Herman, Phys. Rev. B 64, 245407 (2001).

${ }^{6}$ S. Deshpande, S. Patil, S. Kuchibhatla, and S. Seal, Appl. Phys. Lett. 87, 133113 (2005).

${ }^{7}$ F. Zhang, S. W. Chan, J. E. Spanier, E. Apak, Q. Jin, R. D. Robinson, and I. P. Herman, Appl. Phys. Lett. 80, 127 (2002).

${ }^{8}$ I. Kosacki, T. Suzuki, H. U. Anderson, and P. Colomban, Solid State Ionics 149, 99 (2002).

${ }^{9}$ T. Masui, T. Ozaki, K. Machida, and G. Adachi, J. Alloys Compd. 303, 49 (2000).

${ }^{10}$ S. Patil, S. C. Kuiry, S. Seal, and R. Vanfleet, J. Nanopart. Res. 4, 433 (2002).

${ }^{11}$ W. H. Weber, K. C. Hass, and J. R. McBride, Phys. Rev. B 48, 178 (1993).

${ }^{12}$ P. Parayanthal and F. H. Pollak, Phys. Rev. Lett. 52, 1822 (1984).

${ }^{13}$ J. R. McBride, K. C. Hass, B. D. Poindexter, and W. H. Weber, J. Appl. Phys. 76, 2435 (1994). 